\title{
Supernovae driven galactic outflows
}

\author{
Biman B. Nath \\ Raman Research Institute, Sadashivanagar, Bangalore 560080, India \\ email: biman@rri.res.in
}

\begin{abstract}
Outflows from galaxies play a crucial role in the evolution of galaxies and also affect the surrounding medium. The standard scenario of explaining these outflows with the help of supernovae driven wind has recently come under criticism, and other processes such as radiation pressure and cosmic-rays have been invoked. We examine the relative importance of supernovae as the driving mechanism of galactic outflows in light of these competing processes.
\end{abstract}

Keywords. ISM: general- shock waves - galaxies: starburst - intergalactic medium - evolution

\section{Introduction}

Cosmological studies have informed us of the formation of different kinds structures in the universe, primarily through the influence of dark matter, which is the most dominant type of matter in the universe. However, our knowledge of the baryonic component of these structures remain incomplete to a large extent. A proper understanding of the evolution of galaxies requires a study of the complicated physical processes that include star formation, evolution of the ISM and interaction with the intergalactic or intracluster medium. These processes are challenging to study because of the feedback processes that connect one process with another in a complicated manner. These studies require a multi-wavelength approach to observations in order to understand the importance of variation of physical conditions in different phases of gas. On the theoretical side, feedback processes make it difficult to disentangle the effects of different processes. In numerical simulations, the constraint on dynamic range makes it difficult to study together the dynamics of gas inside galaxies as well as its effect on the gas that pervades the intergalactic medium. This constrains one to either focus on the large scale effects after assuming the effects of physical processes working at small scales.

One problem that surfaced in the early studies of galaxy formation and evolution in the cold dark matter cosmologies is that of 'overcooling', as simulations and semi-analytical calculations predicted galaxies which are denser, smaller and contained far too much baryons in the form of stars than was observed. It is now believed that galactic outflows provide an important feedback mechanism for the evolution of galaxies, as well as for the evolution of the intergalactic medium. Outflows can in principle solve the 'overcooling' problem by removing gas from the central region and regulating the process of star formation. It was suggested that low mass galaxies would be more vulnerable to loss of gas through outflows because of their shallow potential well, and therefore the evolution of low mass galaxies would be different from that of massive galaxies (Dekel \& Silk 1986). This could explain the mass-metallicity relation observed in ellipticals, removing the metals preferentially from low mass galaxies and making them metal poor. Outflows were incorporated in the study of metallicity evolution of ellipticals, and the enrichment of the intracluster medium (ICM). After the discovery of metals in the intergalactic medium (IGM), it has been suggested that outflows, especially from low mass galaxies that were abundant at earlier cosmological epochs, could enrich the IGM (Nath \& Trentham 1997). 
More detailed studies of the enrichment of the IGM have found that the prescriptions used for outflow speed and mass loss rate are crucial in understanding the evolution of IGM metallicity (Oppenheimer \& Davé 2006). However, studies aimed at cosmological implications of outflows cannot resolve small scale physical processes and have to depend on different ansatz for outflow parameters. The hot gas in the outflowing matter can also substantially distort the cosmic microwave background through Sunyaev-Zel'dovich effect (e.g, Majumdar et al. 2001) Recently, galactic outflows have also been invoked to solve the problem of reconciling the steep dark matter density profile predicted from simulations and observed profiles with core (e.g., Governato et al. 2010).

However a proper understanding of the effects of outflows requires a detailed knowledge of the mechanisms and the interplay between different physical processes responsible for the outflows. Detailed observations of local galaxies, and spectroscopic studies on high redshift galaxies, show that galactic outflows have far more complicated structure than that of simple steady winds. (Veilleux, Cecil \& Bland-Hawthorn 2005, and references therein). These outflows harbor gas at different phases, from cold and molecular, to warm neutral to partially ionized clouds (that are detected through line radiation or absorption) and which are embedded in a hot medium which shines in x-rays. The standard scenario assumes that multiple supernovae in a star forming galaxy heats up the interstellar medium (ISM), which produces the hot phase of outflows, and that the thermal pressure of the hot gas drives the outflowing gas. The consideration of heating and cooling processes in the ISM heated by supernovae led Dekel \& Silk (1986) to the result that outflows are likely to be more important for low mass galaxies with velocity dispersion $\leqslant 100 \mathrm{~km} \mathrm{~s}^{-1}$, i.e., dwarf galaxies. Since the temperature of the hot phase of outflowing gas is found to be $\sim 1 \mathrm{keV}$ (Martin 2005), this implies a near constant speed of the outflowing gas, of order the thermal speed. Chevalier \& Clegg (1985) considered supersonic outflowing wind from starbursts such as M82 (ignoring the effect of gravity), and suggested a outflow speed in excess of $1000 \mathrm{~km} \mathrm{~s}^{-1}$. Wang (1995) included the effect of dark matter potential and found that gravity can considerably slow down the wind speed. A number of simulations have also studied the dynamics of outflowing gas, especially in comparison with the observations of M82 (e.g., Strickland \& Stevens 2000).

There are however a number of problems with this scenario of thermal pressure driven outflows. Firstly, there is a problem of the survival of the cold/warm clouds in the hot gas. Observations find these clouds at large distances (several kpc) from the central star forming regions of the galaxy. Assuming thermal speed of a $1 \mathrm{keV}$ gas, the time taken by the clouds to reach such distances is $\sim 10 \mathrm{Myr}$. This long travel time makes it difficult for the clouds to survive against several instabilities at work, e.g., Kelvin-Helmholtz instability or evaporation by thermal conduction, which have a time scale of order $\leqslant 1$ Myr (Marcolini et al. 2005). Secondly, recent observations show a correlation between the maximum speed of cold/warm clouds (at $10^{4} \mathrm{~K}$ ) embedded in the hot medium and the galaxy rotation speed (Martin 2005). Also, observations of Lyman break galaxies at high redshift show that the outflow speed is correlated with the star formation rate (Shapley et al. 2003), as well as with the reddening due to dust. Ferrara and Ricotti (2006) found that the observed correlation of outflow speed with star formation rate is difficult to explain in the standard SN-driven wind scenario.

Murray et al. (2005) pointed out the importance of radiation pressure in outflows and showed that it can be comparable to the ram pressure of the hot gas on the cold clouds. They worked out the dynamics of radiatively driven shells of gas and dust, and Martin (2005) found that this model explains the observations of cold clouds better than the thermal pressure scenario. Nath \& Silk (2009) discussed a hybrid model of outflows from 
Lyman break galaxies with ram and radiation pressure. In this scenario the radiation pressure from massive stars in a stellar population pushes a shell of gas and dust out to a large distance, and then the ram pressure from SNe heated gas pushes it further. The shell fragments due to the acceleration and can explain the existence of clouds at large distances (see also Murray et al. 2011).

\section{Ram and radiation pressure}

The effect of radiation pressure for a disk galaxy can be estimated in terms of an Eddington parameter, $\Gamma_{0}=\kappa I /(2 c G \Sigma)$, where $\kappa$ is the opacity of a mixture of dust and gas (in the units of $\mathrm{cm}^{2} \mathrm{~g}^{-1}$ ), $I$ and $\Sigma$ are the surface brightness and surface mass density of the disk respectively. Recently, Sharma et al. (2011) considered a general case of wind driven by radiation pressure on dust grains from a disk galaxy and calculated the outflow speed as a function of the galaxy rotation speed. They argued that although the details of the wind structure depends on various parameters, the terminal speed of the outflowing gas can be estimated in terms of the parameters near the disk by using the Bernoulli equation. They considered a halo with Navarro-Frenk-White density profile, a spherical bulge and a disk with constant surface density, and assumed an instantaneous burst of star formation. They found that the minimum value of the Eddington parameter $\Gamma_{0}$ for outflows to escape the virial radius of a halo to be $\sim 2$, which corresponds to a mass to light ratio (in the solar units) of $\Upsilon_{B} \leqslant 0.04$ (for typical NFW parameters of a galaxy, and for $\left.\kappa \sim 200 \mathrm{~cm}^{2} \mathrm{~g}^{-1}\right)$. Although this is much smaller than the observed values of disk galaxies $\left(\Upsilon_{B} \sim 1.5\right)$, starburst galaxies can have a much lower value. They also found that the ratio of the outflow terminal speed to the galaxy rotation speed is $\sim 2-4$ for a period of $\sim 10 \mathrm{Myr}$, consistent with observations. Also, simulations of IGM enrichment claim to better match with data when they use a terminal speed of order three times the galactic rotation speed (Oppenheimer \& Davé 2006).

This result was confirmed with a 2-D hydrodynamic simulation, which further enabled one to study the structure of the wind in the presence of angular momentum of the gas lifted from a rotating disk (Chattopadhyay et al. 2012). They found that the combined effect of gravity and radiation pressure from an exponential, rotating disk can drive the outflowing gas out to a distance of $\sim 5 \mathrm{kpc}$ in $\sim 40 \mathrm{Myr}$ for typical galactic parameters. The outflow speed was found to increase rapidly for $\Gamma_{0} \geqslant 1.5$. The wind was seen to develop a conical structure because of the angular momentum of the gas, and the rotation speed of the wind was less than the galaxy rotation speed. The structure of the rotating wind was similar to that in M82, and another such example has been recently observed by Vogt et al. (2013) in the case of HCG16.

In order to compare the relative roles of ram and radiation pressure in driving the clouds embedded in outflows, Sharma \& Nath (2012) considered outflows from disk galaxies with continuous star formation, and included the effects of gravity due to an exponential disk, a spherical bulge and dark matter halo. They presented the results in the parameter space of the star formation rate (SFR) and the rotation speed of galaxies, and in terms of the ratio of outflow speed in the units of the galaxy rotation speed, so that the results could be compared with data from outflows. According to their results, the dynamics of clouds in outflows from galaxies with rotation speed $v_{c} \leqslant 200 \mathrm{~km}$ $\mathrm{s}^{-1}$ and $\mathrm{SFR} \leqslant 100 \mathrm{M}_{\odot} \mathrm{yr}^{-1}$ is dominated by ram pressure, and radiation pressure becomes more important in more massive galaxies and with with larger SFR. Further, they found that the ratio of mass loss rate in the wind and star formation rate scales as $v_{c}^{-1} \Sigma_{g}^{-1}$, where $\Sigma_{g}$ is the gas column density of the disk. Recent simulations confirm such a 
division in the regimes of thermal and radiation pressure dominated outflows in terms of galactic mass, and the dependence of mass loss rate with the galactic rotation speed.

A crucial parameter in this calculation is the assumption of the speed of the hot gas in the outflows. As mentioned earlier, this has been assumed to be either of order thermal speed appropriate for a gas with temperature $\sim 0.5-1 \mathrm{keV}$ ( for which the isothermal speed is $\sim 300-400 \mathrm{~km} \mathrm{~s}^{-1}$ ), or a larger speed corresponding to a supersonic case. It therefore becomes important to study the transonic solutions of the hot gaseous wind, especially in the presence of both thermal and radiation pressure. There is also the possibility of radiation pressure from an accreting, luminous black hole in the central regions of massive galaxies. Sharma \& Nath (2012) considered steady spherical wind driven by energy, mass and momentum injection processes that are confined to a central region of size $\sim 200 \mathrm{pc}$. One can write a general equation for the transonic wind in terms of the Mach number, which reduces to the Chevalier \& Clegg (1985) solution in the case of zero gravity.

Sharma \& Nath (2012) showed that the dynamics of winds is determined by the interplay of three velocity scales: (a) $v_{*} \sim(\dot{E} / 2 \dot{M})^{1 / 2}$ is related to the starburst activity, with $\dot{E}$ and $\dot{M}$ as the energy and mass injection rate in the central region; (b) $v_{b h} \sim\left(G M_{b h} / 2 R\right)^{1 / 2}$ reflects the gravitational effect of a central black hole on gas in the central region, and (c) $v_{s}=\left(G M_{h} / 2 \mathcal{C} r_{s}\right)^{1 / 2}$, which is related to the galactic rotation speed for an NFW halo for halo mass $M_{h}$, where $r_{s}$ is the scale length and $\mathcal{C}$ is a function of the concentration parameter. The values of $v_{*}$ range from $\sim 200-500$, depending on the efficiency of supernovae to deposit energy in the ISM, with a larger value being appropriate for starburst galaxy, and lower values for Milky Way type galaxies with quiescent SFR. This follows naturally from the definition of $v_{*}$ that $v_{*} \sim 560 \sqrt{\alpha} \mathrm{km} \mathrm{s}^{-1}$, where $\alpha$ denotes the efficiency of SN energy thermalisation, and the two limiting values of $v_{*}$ mentioned above follow from the assumption of $\alpha \sim 0.1-1$.

The generalized formalism then allows one to determine the terminal velocity of outflows to be $\left(4 v_{*}^{2}+6(\Gamma-1) v_{b h}^{2}-4 v_{s}^{2}\right)^{1 / 2}$, where $\Gamma$ is the ratio of force due to radiation pressure to gravity of the central black hole. The hot wind speed in the low to intermediate mass galaxies was determined to be in the range $~ 400-1000 \mathrm{~km} \mathrm{~s}^{-1}$. On the contrary, massive galaxies with central black holes radiating at Eddington limit have outflows with speed in excess of $1000 \mathrm{~km} \mathrm{~s}^{-1}$. Observations of terminal speed of outflows can therefore be a useful diagnostic of whether or not it is driven by supernovae or active galactic nuclei (AGN). This limiting speed of $\sim 1000 \mathrm{~km} \mathrm{~s}^{-1}$ is supported by recent observations (Tremonti et al. 2007; Sturm et al. 2011).

Sharma \& Nath (2012) further showed that winds from quiescent star forming galaxies cannot escape for intermediate size galaxies $\left(10^{11.5} M_{\odot} \leqslant M_{h} \leqslant 10^{12.5} M_{\odot}\right)$, and that these galaxies should harbor a large amount of gas in their halos. This is consistent with the observations of Tumlison et al. (2011) in which halos of galaxies in this mass range were reported to contain a large amount of hot gas.

They derived a ratio $\left[2 v_{*}^{2}-(1-\Gamma) v_{b h}^{2}\right] / v_{c}^{2}$ which is proportional to the amount of gas lost through the winds. The behavior of this ratio with galactic mass therefore determines the ability of galaxies to expel or retain their gas reservoir, and this function can be evaluated by assuming an appropriate relation between the central black hole mass and the galactic mass, and an appropriate opacity of dust grains in the infrared (K) band. It is interesting that the value of this ratio has a minimum at the galactic mass scale of $\sim 10^{12-12.5} M_{\odot}$, which signifies the change in regime of supernovae to AGN domination in outflow properties. Used with a simple chemical evolution model of galaxies, they showed that this result is consistent with the observed trend of stellar mass to galactic 
mass ratio, and that the above mentioned range of hot gas wind (400-1000 $\left.\mathrm{km} \mathrm{s}^{-1}\right)$ can explain the observed scatter in the observed ratio of stellar to halo masses.

\section{Cosmic ray driven wind}

Besides the effect of thermal and radiation pressure, another important physical processes that has been invoked to be responsible for driving outflows is that involving cosmic rays. It is believed that when cosmic rays stream with respect to the ambient medium, they excite Alfvén waves on which they scatter, and consequently limit the effective bulk speed of cosmic rays. The damping of Alfvén waves excited by streaming cosmic rays can lead to the heating of the ISM and subsequently to outflows. Recently, a detailed numerical simulation that includes the physics of cosmic ray streaming, it was found that this process can drive galactic winds from galaxies with halo masses $\leqslant 10^{11} \mathrm{M}_{\odot}$ (Uhlig et al. 2012). This process also suppresses the star formation rate, particularly in dwarf galaxies $\left(10^{9} \mathrm{M}_{\odot}\right)$, in which $\sim 60 \%$ of the baryonic matter is expelled, with a mass loss rate reaching a factor of 5 more than the star formation rate.

These results are also supported by analytical estimates done with the help of the Bernoulli equation, as in the case of radiation pressure driven winds. Furthermore, the simulations showed that in higher mass galaxies, the winds develop a bi-conical structure. It is possible that the early galactic halos, which were mostly low mass galaxies, were affected by cosmic ray pressure driven winds, since radiation pressure would have been of less importance because of lack of metals required to produce dust grains. Also, there are a few examples of galactic outflows which occupy a region of the parameter space of SFR and galactic rotation speed, as in Sharma \& Nath (2012), that are neither explained by thermal or radiation pressure adequately, with very low SFR $\left(\leqslant 0.1 \mathrm{M}_{\odot} \mathrm{yr}^{-1}\right)$ and low galactic rotation speed $\left(\leqslant 60 \mathrm{~km} \mathrm{~s}^{-1}\right)$. Interestingly, the examples simulated by Uhlig et al. (2012) fall in this range of parameters, and it would be curious to study such cases with more detailed simulations in the future.

\section{Threshold condition for supernovae driven outflows}

Another important problem to study is the conditions required to launch galactic outflows from disk galaxies. Although it is believed that in general galaxies with high SFR excite such outflows, Heckman (2002) argued that the determining condition is related to the surface density of SFR, and not the average SFR in the galaxy. He found a threshold SFR of $\sim 0.1 \mathrm{M}_{\odot} \mathrm{yr}^{-1} \mathrm{kpc}^{-2}$ for the existence of galactic winds. Another set of observations, aimed at studying the extraplanar gas in edge-on disk galaxies, found a minimum value of energy injection that were required for the existence of synchrotron emitting gas. The threshold surface density of energy injection is $\sim 10^{-4} \mathrm{erg} \mathrm{s}^{-1} \mathrm{~cm}^{-2}$ (Dahlem et al. 1995). Tüllmann et al. (2006) further considered the conditions for the existence of X-ray, radio and far-infrared emission from extended halo gas in disk galaxies, and found the minimum energy injection rate to be $\sim 10^{-3} \mathrm{erg} \mathrm{s}^{-1} \mathrm{~cm}^{-2}$.

Recently, it has been argued that these threshold conditions can be understood from the requirement of multiple $\mathrm{SNe}$ driven superbubbles to compensate against radiative loss and be able to break out of the disk with sufficient Mach number in order for the hot interior gas to reach a considerable height above the disk (Roy et al. 2013). The basic argument can be illustrated with the help of a simple estimate. Consider a SN remnant, which enters the radiative phase when the shock speed becomes $\sim 70 \mathrm{~km} \mathrm{~s}{ }^{-1}$, corresponding to a post-shock temperature of $\sim 10^{5} \mathrm{~K}$, where the cooling function peaks. We denote this epoch by $t_{\text {rad }} \sim 3 \times 10^{5}$ yr $E_{51}^{1 / 3} n^{1 / 3}$, where $E=10^{51} E_{51}$ erg is the typical 
energy of a SN, and $n$ is the particle number density of the ISM in the units of $\mathrm{cm}^{-3}$. The corresponding radius is $R_{\text {rad }} \sim 50 \mathrm{pc} E_{51}^{1 / 3} n^{1 / 3}$. Writing the volume rate of SN as $\nu_{S N}$, the condition that multiple SN can inject enough energy for the superbubble not to stall after reaching the radiative phase can be written as, $(4 \pi / 3) R_{\text {rad }}^{3} t_{\text {rad }} \nu_{S N} \geqslant 1$, which translates to $\nu_{S N}>6 \times 10^{-12}\left(n / E_{51}\right)^{4 / 3} \mathrm{SNe} \mathrm{yr}^{-1} \mathrm{pc}^{-3}$. The surface density of SNe can be found by multiplying this by the scale height (which can be shown to be comparable to the size of the bubble in the disk plane during the break out phase), and this leads to $6 \times 10^{-4} \mathrm{SNe} \mathrm{yr}^{-1} \mathrm{kpc}^{-2}$, for a scale height of $100 \mathrm{pc}$. Using Salpeter IMF, and considering stellar masses in the range of 1-100 $\mathrm{M}_{\odot}$, the corresponding surface density of SFR becomes $\sim 0.1 \mathrm{M}_{\odot} \mathrm{yr}^{-1} \mathrm{kpc}^{-2}$, consistent with observations.

Roy et al. (2013) has worked out the dynamics of superbubbles triggered by multiple SNe in a disk galaxy, first analytically and then with hydrodynamic simulations, and they found that this simple estimate is supported by detailed calculations. Furthermore, they studied the clumping and fragmentation of the superbubbles during its evolution, and found that thermal instability plays an important role in clumping of the shell material, and in subsequent seeding of Rayleigh-Taylor instability. Without the aid of thermal instability, Roy et al. (2013) showed that the clumping due to Rayleigh-Taylor instability usually takes place at a time scale that is longer than the main sequence life time of $\mathrm{O}$ stars, the main source of ionizing radiation. This problem is therefore important not only for the hot interior gas to be released into the halo region, but also for the escape of ionizing radiation from massive stars. The latter issue is relevant for the problem of reionization of the IGM.

\section{Summary}

Although thermal pressure of supernovae heated gas remains the dominant mechanism for driving outflows from galaxies, other physical processes such as radiation and cosmic ray pressure can also play important roles. Recent analytical and simulations show that radiation pressure becomes important for massive galaxies either with high SFR or with an AGN component. The signature of AGN driven winds can be diagnosed from the outflow speed which in this case can exceed $1000 \mathrm{~km} \mathrm{~s}^{-1}$. Cosmic ray pressure can be important for low mass galaxies. Intermediate sized galaxies with quiescent SFR are predicted to contain a large amount of gas in their halo. Also, consideration of radiative cooling is important to understand the threshold conditions for excitation of galactic winds from disk galaxies.

\section{References}

Bisnovatyi-Kogan, G. S. \& Silich, S. A. 1995, Rev Mod Phys, 67, 661

Chattopadhyay, I., Sharma, M., Nath, B. B., \& Ryu, D. 2012, MNRAS, 423, 2153

Chevalier, R. A. \& Clegg, A. W. 2009, Nature, 317, 44

Dekel, A. \& Silk, J. 1986, ApJ, 303, 39

Ferrara, A. \& Ricotti, M. 2006, MNRAS, 373, 571

Governato, F. et al. 1986, Nature, 463, 203

Heckman, T. M. 2002, in: L. S. Mulchaey \& J. Stocke (eds.), ASP Conf. Ser. 254, Extragalactic Gas at Low Redshift (San Fransisco, CA: ASP), p. 292

Majumdar, S., Nath, B. B., \& Chiba, M. 2001, MNRAS, 324, 537

Marcolini, A. Strickland, D. K., D'Ercole, A., Heckman, T. M., \& Hoopes, C. G. 1986, MNRAS, 362,626

Martin, C. L. 2005, ApJ, 621, 227

Murray, N., Quataert, E., \& Thompson, T. A. 2005, ApJ, 618, 569 
Murray, N., Ménard, B., \& Thompson, T. A. 2011, ApJ, 735, 66

Nath, B. B. \& Trentham, N. 1997, MNRAS, 291, 505

Nath, B. B. \& Silk, J. 2009, MNRAS, 396, L90

Oppenheimer, B. D. \& Davé, R. 2006, MNRAS, 373, 1265

Roy, A., Nath, B. B., Sharma, P., \& Shchekinov, Y. 2013, arxiv:1303.2664

Shapley, A. E., Steidel, C. C., Pettini, M., \& Adelberger, K. L. 2003, ApJ, 588, 65

Sharma, M., Nath, B. B., \& Shchekinov, Y. 2011, ApJL, 763, 27

Sharma, M. \& Nath, B. B. 2012, ApJ, 750, 55

Sharma, M. \& Nath, B. B. 2013, ApJ, 763, 17

Strickland, D. K. \& Stevens, I. R. 2000, ApJ, 314, 511

Sturm, E. et al. 2011, ApJL, 733, 16

Tremonti, C. A., Moustakas, J., \& Diamnond-Stanic, A. M. 2007, ApJL, 663, 77

Tumlinson et al. 2011, Science, 334, 948

Uhlig, M., Pfrommer, C., Sharma, M., Nath, B. B., Enßlin, T. A., \& Springel, V. 2012, MNRAS, 423,2374

Veilleux, S., \& Gerald, C. Bland-Hawthorn, J. 2005, ARAA, 43, 769

Wang, B. et al. 1995, ApJ, 444, 590

\section{Discussion}

WANG: One possibility is that condensations can be important when seeds of cool gas clouds are available which can be driven out by radiation pressure, for example.

NATH: Yes, this possibility needs to be explored.

Hensler: Two comments: 1. Galactic winds models usually do not take the external gas into account, e.g. Hot holo-gas, surrounding gas disks in star burst galaxies, both hampering the outflow. 2. HVC should be self-gravitating according to their mass. This suppresses Rayleigh Taylor instability. In addition when passing through hot halo-gas, HVCs experience thermal conduction. Vieser \& Hensler (2007, A \& A, 475, 251) have shown that this thermal conduction suppresses Kelvin Helmholtz instability so that such HVCs can survive for 100 Myrs and longer.

NATH: Thank you for the comments. Perhaps the issue of cloud survival in hot wind needs more work.

NTORMOUsI: Regarding the growth of RT and KH instabilities on the surfaces of cold clumps embedded in a hot medium. What is the relationship between the size of the clouds and the mean free path of the particles in the hot gas? (a large mfp would mean no hydro instabilities).

NATH: The mean free path is smaller than typical cloud sizes.

SINGH: If supernova outflows can create a global thermal equilibrium in scales as large as intracluster medium of galaxy clusters, can the mass outflow alter the NFW profile of these relaxed halos?

NATH: These outfows are thought to affect the dark matter profile at very small scales $(<1 \mathrm{kpc})$ but not at larger scales. 